\title{
Polar auxin transport and patterning: grow with the flow
}

\author{
Ben Scheres ${ }^{1}$ and Jian $\mathrm{Xu}$ \\ Department of Biology, Utrecht University, $3584 \mathrm{CH}$ Utrecht, The Netherlands
}

Particularly in the fall, plant leaves demand attention as examples of the beauty of nature. Around that time, anyone can pick them up and have a close look at the variety in venation patterns. For a considerable time, biologists and mathematicians went one step farther, trying to find regularities in these patterns and rules behind them. Vein organization can be classified in several general patterns characteristic of a particular plant (Fig. 1A-D), suggesting that genetic input into the patterning system can be responsible for predictable gross changes in venation networks. However, close inspection reveals that there is considerable variation in the venation pattern in individual leaves of many plants. This reveals a large stochastic element in the mechanism by which veins are formed. In this issue of Genes \& Development, Scarpella et al. (2006) provide new insights into how genetic and stochastic vein patterning processes might be related, but also connect the initiation of venation patterns to recent models of other auxin-dependent patterning processes in shoots and roots.

\section{Venation networks: models and observations}

Observations at the cellular level indicate that vein progenitor cells ("pre-procambial" cells) become incorporated into veins by selection from equivalent subepidermal leaf cells. During this process, pre-procambial cells divide and elongate along a common axis, which is essential for the formation of a continuous vein network that can carry out its various transport roles. Therefore, any model to explain venation network formation will have to explain how narrow rows of precisely connected cells can be specified from a uniform field of cells. Then, it will have to explain how different regularities can be "programmed" to give rise to the characteristic venation classes. Lastly, it will have to deal with the observed variability in final patterns.

Over the years, two major models have been proposed to start explaining vein patterning, both of which incorporate self-organizing properties that can give rise to the variable features of venation networks. Model 1 elabo-

${ }^{1}$ Corresponding author.

E-MAIL b.scheres@bio.uu.nl; FAX 31-30-251-3655.

Article and publication are at http://www.genesdev.org/cgi/doi/10.1101/ gad.1426606. rates on Turing's reaction-diffusion principle (Turing 1952). The basic idea is that the autocatalytic production of an activator triggers a faster diffusing inhibitor, which keeps a new activator at a distance. Depending on parameters, this system can form connected or disconnected net-like pre-patterns (Fig. 1E,F), as well as freely ending networks if the activator in addition removes its own substrate (Fig. 1G; Meinhardt 1976, 1984). Model 2, the "canalization hypothesis," specifically proposes the plant growth substance auxin as a patterning agent. This model is rooted in observations on the vein-inducing capacity of auxins and proposes that positive feedback between auxin flow through a cell and the capacity of that cell to transport auxin leads to preferred conduits, which will differentiate as veins (Mitchison 1980; Sachs 1981, 1991; Rolland-Lagan and Prusinkiewicz 2005).

Since the model organism Arabidopsis thaliana has allowed the collection of venation mutants within a single species, a lively debate has taken shape in the literature on whether such mutants allow distinguishing between models 1 and 2 . A critical distinction between the two models is that discontinuous, "patchy" initial specification of venation networks is allowed by reaction-diffusion models but not by canalization models. It is therefore not surprising that observations of discontinuous and unconnected regions of vein specification, so-called vascular islands, were proposed to refute canalization models in favor of reaction-diffusion mechanisms for vein precursor specification (Carland et al. 1999; Deyholos et al. 2000; Koizumi et al. 2000). However, discontinuities in the initiation of vein pattern can only be defined by the earliest markers available.

In this issue of Genes \& Development, Scarpella et al. (2006) use a functional fusion of GFP with one of the Arabidopsis pin-formed (PIN) transmembrane polar auxin transport proteins, PIN1. The PIN1 protein marks incipient vein cells earlier than any other pre-procambial marker described so far (Scarpella et al. 2006), as well as cell polarity (Gälweiler et al. 1998). Thus, PIN1:GFP monitoring can provide a direct observation of the basic mechanism proposed to act in the canalization hypothesis. Excitingly, the findings reported by Thomas Berleth's group (Scarpella et al. 2006) provide a strong case for canalization mechanisms operating during vein development. 

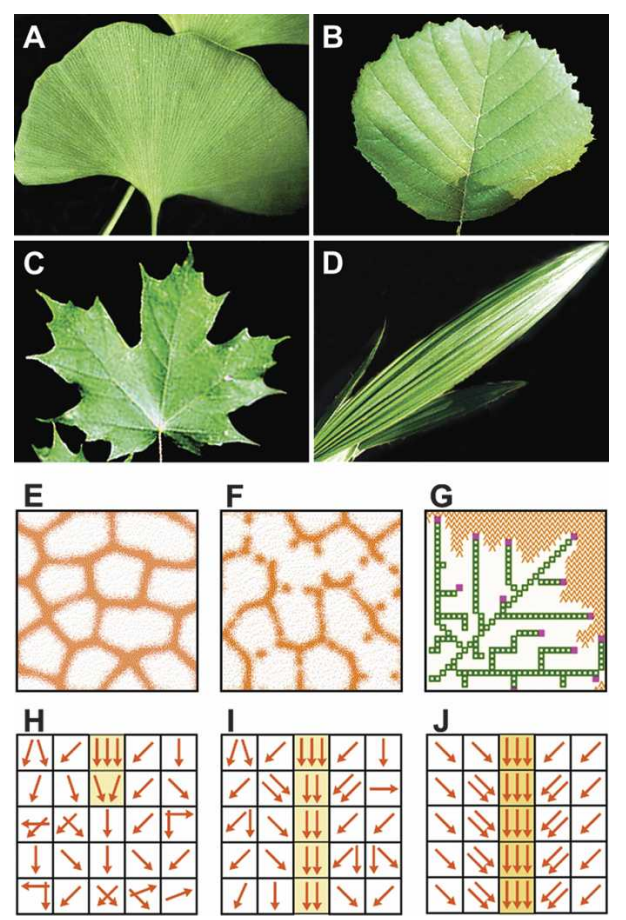

I
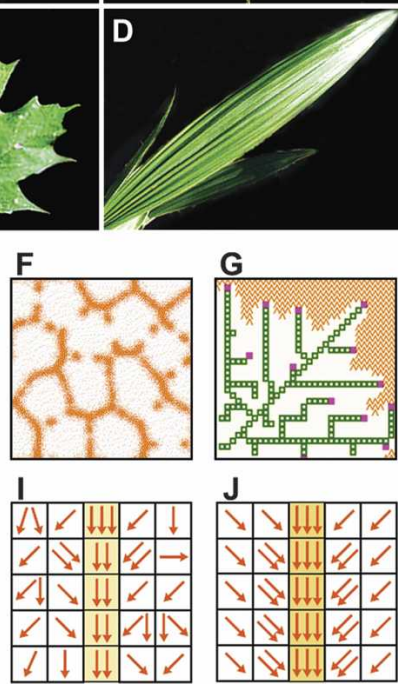

G
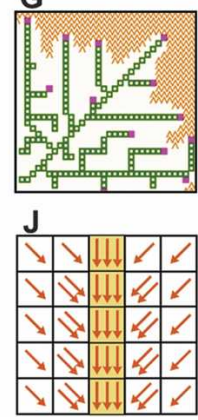

Figure 1. $(A-C)$ Leaves with reiterative branching patterns. $(D)$ Parallel branching. $(E-G)$ Distribution of activator and inhibitor after simulation of reaction-diffusion models. Activator inhibitor system with different parameter settings yields connected $(E)$ or disconnected $(F)$ patterns. $(G)$ Inclusion of substrate depletion yields open vein network. $(H, I)$ Fluxes in canalization model. Initially, stochastic accumulation of activating molecules $(H)$ induces polarized transport and differentiation of conduits $(I, J)$. $E$ and $F$ are modified from Berleth (2000) (C 2000, with permission from Elsevier), $G$ is modified from Meinhardt et al. (1998) (C 1998, with permission from World Scientific Publishing Co. Pte. Ltd., Singapore), and $H-J$ are modified from Scheres and Berleth (1998) (C 1998, with permission from Elsevier).

\section{A combination of two principles underlie vein patterning}

In a technical tour de force that involved monitoring of expression and polarity of the PIN1:GFP fusion ("PIN1" from here on) in numerous early leaf primordia, the following observations were made (Fig. 2A-C; Scarpella et al. 2006): (1) The first expression of PIN1 is epidermal with a convergence point containing cells with opposite PIN1 polarity at the leaf primordium tip. This position foreshadows the formation of the midvein. (2) The formation of branches of the midvein is foreshadowed by new epidermal convergence points at the leaf margin of the growing leaf primordium, again defined by cells with opposing PIN1 localization. (3) New vein extensions start out as free ends (toward pre-existing veins) and connect to previously formed veins with PIN1 polarity directed toward them. (4) PIN1 localization identifies closed veins as composite expression domains with opposite polarity, connected by a single internal bipolar cell. Importantly, such expression domains start out as free-ending veins. (5) PIN1 expression domains start out as wide domains that subsequently narrow down.
These observations suggest a "leaf-margin guided" venation model composed of two separate processes: (1) Specification of a PIN1 convergence domain and auxin accumulation at the convergence point lead to elevation of PIN1 expression and polarization correlated with the gradual selection of a narrow strand of pre-procambial cells (Fig. 2A,B). (2) Pre-existing veins polarize surrounding cells, which leads to "backward" recruitment of preprocambial cells that meet and connect to form a closed network (Fig. 2B). The second principle is used over and over again to specify the many higher-order veins in the network, and bipolar cells are the likely meeting points of these growing vein ends (Fig. 2C, arrowhead). The last added veins can remain "open" because the process is halted at the time when mesophyll cells, the alternative choice for internal leaf precursor cells, start to differentiate (Scarpella et al. 2004).

Earlier observations had already implicated auxin accumulation and the regulation of this process by polar auxin transport in vein formation, but these experiments relied on late markers of venation (Mattsson et al. 1999). Using PIN1 as the new early pre-procambial marker, these correlations could be established at earlier stages of the pre-procambial selection process (Scarpella et al. 2006). Auxin application in lanolin paste led to more epidermal PIN1 convergence points, but did not interfere with self-restriction of the size of internally projected procambial PIN1 expression domains. The polar auxin transport inhibitor NPA, on the other hand, provoked more convergence points but also delayed self-restric-

A
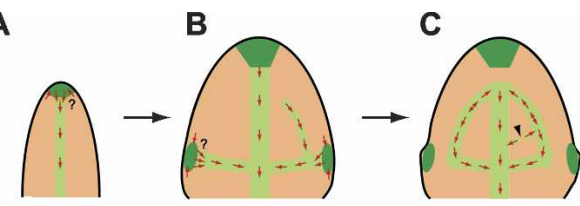

D

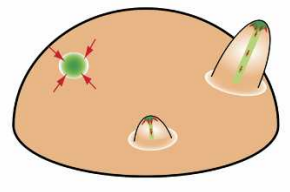

E

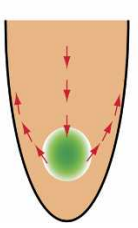

Figure 2. $(A-C)$ Model for venation by Scarpella et al. (2006). Three stages of leaf primordia are shown. Polar PIN1 expression and presumed auxin flow (arrows), epidermal convergence points (dark green), and pre-procambial cells (light green). (A,B) The positions of the first vein and its branch points are guided by epidermal convergence points. New veins are added onto existing ones with PIN1 polarity directed to the older vein. $(C)$ Loops in the network are formed when veins join (arrowhead), after which two domains with opposite PIN1 polarity may be joined by a bipolar cell (double arrows). (D) Specification of leaf primordia at the surface of the shoot apical meristem also involves epidermal convergence points of PIN1 polarity correlated with auxin maxima, which are thought to generate leaf spacing (phyllotaxis) through a self-organizing process. $(E)$ Root primordia contain auxin maxima correlated to PIN proteins that have a different polarity: toward the maximum in central cells and away from the maximum in epidermis. 
tion. Hence, both auxin accumulation and auxin transport influence the currently earliest detectable stages of vein formation.

Because the strongest case against canalization was, up until now, made by the "vascular island" mutants, the authors went on and studied pre-procambial cell selection as monitored by PIN1 accumulation and polarity in one of the strongest vascular network mutants, van3. In support of canalization-related models, early leaf vascular development as marked by PIN1 is initially continuous in a van3 mutant background. This continuity "breaks up" when later markers are examined. "Islands" therefore appear to result from inappropriate stabilization of cell fate, which isolates previously connected pre-procambial cells. Interestingly, VAN3 encodes an auxin-responsive adenosine diphosphate (ADP)-ribosylation factor-guanosine triphosphatase (GTPase)-activating protein (ARF-GAP) (Koizumi et al. 2005). Although initial experiments link VAN3 to auxin sensitivity rather than polar auxin transport, ARF-mediated vesicle trafficking has been shown to be important for membrane recycling of PIN proteins (Geldner et al. 2003; Xu and Scheres 2005). It is therefore possible that vascular island formation results from inappropriate regulation of auxin sensing or polar auxin transport.

Taken together, the data provided by Scarpella et al. (2006) are entirely consistent with the canalization hypothesis, but they add an extra factor: Initiation of branching can be controlled at the level of the specification of PIN1 convergence points. While the canalization concept is reinforced as the stochastic mechanism that specifies vein tracks in a field of growing cells, the question of the different classes of venation patterns in nature can now be related to genetic factors that contribute to the initiation of PIN1 convergence points and the associated auxin response maxima that suggest auxin accumulation at these points.

With these new insights, variations in venation pattern in different species can now be viewed as a different balance between self-organizing and genetically determined positioning of epidermal PIN1 convergence points.

How definitive is the evidence proposed by Scarpella et al. (2006) with respect to formal models of vein formation? One can play the devil's advocate, suggesting that the key argument-continuity of specificationstill rests on PIN1 as the earliest available marker, and that the identification of an even earlier and more patchy marker for procambial specification could reverse the argument. While this cannot be excluded until each step of the pre-procambial specification process is known, it seems unlikely that an alterative scenario can easily explain the potent venation response to external auxins (Sachs 1981), correlations of venation patterns with auxin (response) patterns (Mattsson et al. 1999; Aloni et al. 2003) the auxin-inducibility of PIN1 expression (Vieten et al. 2005), the requirement for polar auxin transport in vein patterning (Mattsson et al. 1999; Sieburth 1999), and the consistency between the experimental data of Scarpella et al. (2006) and recent simula- tions of leaf venation using canalization models (Rolland-Lagan and Prusinkiewecz 2005). Moreover, a recent study that establishes a causal relationship between PIN polarity and direction of polar auxin transport (which hitherto was inferred by correlation) (Wisniewska et al. 2006) elevates the continuous PIN1 domains from a mere marker into a falsifiable predictor of the canalization model. A remaining open question at this point is how the epidermal PIN1 convergence points connect to the polar expression domains of the internal tissues (Fig. 2A,B, question marks).

\section{Auxin accumulation in plant patterning: generalizations to roots and shoots}

Evidence for "auxin maxima" as essential elements for patterning in diverse developmental contexts has accumulated over the last decade. Indications for the presence of auxin maxima, their dependence on polar auxin transport, and evidence for their importance in cell specification and cell polarity were first obtained in the root tip (Sabatini et al. 1999). In roots, the activity of PIN proteins has been shown to be instrumental for the localization of auxin maxima and for the development of the root primordium (Friml et al. 2002; Blilou et al. 2005). The use of more sensitive reporters has expanded the notion of PIN-dependent auxin maxima to growing primordia in the shoot apex (Friml et al. 2003; Reinhardt et al. 2003; Heisler et al. 2005). Whether occurring in roots or shoots, these auxin maxima find themselves at positions where cells with opposing PIN polarity meet. However, the PIN protein localization pattern is different, as PIN proteins converge in shoot maxima and diverge in root maxima (Fig. 2D,E). Regardless of this difference, both maxima "organize" organ formation (root growth and patterning, and shoot primordium outgrowth) and are or become connected to vascular tissue. And now, the new study of Scarpella et al. (2006) has added to this list an intriguing set of PIN convergence domains at the leaf margin that organize vascular development (Fig. 2A-C). The latter study suggests that intricate feedback between auxin accumulation and cell polarity (that defines PIN localization) plays a role in setting up the venation pattern. Can we generalize here and identify a common mode for pattern formation in diverse organs where (1) feedback mechanisms establish an auxin maximum and (2) the maximum, dependent on context-derived competence factors, organizes major aspects of subsequent development? For this we first turn to shoots, then to roots.

The application of auxins to polar auxin transportdefective inflorescence apices provided evidence that auxin accumulation drives the definition of new leaf-like primordia in shoot apical meristems (Reinhardt et al. 2000). Subsequent analysis revealed that sites of new and outgrowing primordia revealed PIN convergence domains in a single cell layer, the epidermis, and related auxin response maxima (Friml et al. 2003; Reinhardt et al. 2003). At that stage, it was realized that auxin accumulation toward existing primordia could deprive 
surrounding regions from auxin, thus creating a zone of inhibition that could explain the geometrically highly ordered positioning of primordia. Recently, a series of simulations that derive shoot meristem maxima from polar auxin transport properties has been published in an attempt to explain phyllotaxis (de Reuille et al. 2006; Jonsson et al. 2006; Smith et al. 2006). Different primary assumptions have been made to enable in silico generation of maxima in a pattern that matches the emergence of leaf primordia in spirals or whorls. Among them, the most conspicuous "rules" were to allow a cell to position PIN proteins toward neighboring cells with higher auxin levels (Jonsson et al. 2006) and to make PIN1 levels auxin dependent (Smith et al. 2006). While several additional rules were needed to make the different phyllotaxis models run, these two appeared to be powerful in creating the patterns observed in nature. Intriguingly, polarity reversals at the edges of flower primordia have been observed (Heisler et al. 2005), and the combination of experimental and modeling evidence makes a case that these might be changes caused by the proposed (as yet unknown) mechanism of polarizing according to a neighbor's concentration. It is remarkable that polarization of PIN1 according to auxin concentration is closely connected now to both phyllotaxis and venation patterns.

Recently, it has been investigated whether laser ablation-induced shifts of the auxin maximum in the root tip (that lead to the respecification of the cell types associated with the maximum) affect PIN expression and polarity (Xu et al. 2006). Remarkably, no PIN polarity changes were observed around the shifting auxin maximum, but conspicuous effects on PIN expression were noted. However, PIN polarity in the root could change dramatically in the absence of transcription factors that are required for tissue identity. In another study, the auxindependent transcription factors of the PLETHORA family (Aida et al. 2004) were shown to be required for the expression of PIN proteins in specific domains (Blilou et al. 2005). These data indicate that transcription factors can stabilize cell polarity and make it only indirectly dependent on auxin distribution. Furthermore, they show that transcription factors can dictate the amount and polarity of PIN proteins differently in different cell types. This may explain how a single auxin maximum is maintained in the root tip and how new maxima, which correlate with new PIN expression domains and auxin accumulation patterns in lateral roots (Benkova et al. 2003), may emerge later by "freeing up" feedback mechanisms when tip-enriched transcription factors reach lower levels.

Despite obvious differences between vein patterning, leaf primordium outgrowth, and root cell type specification, we can now begin to compare patterning mechanisms during venation and shoot and root primordium definition. All these processes are associated with organizing PIN convergence and auxin accumulation points, and with feedback of auxin accumulation on PIN polarity and/or expression. Important remaining questions are what molecular mechanisms create PIN convergence points in root and shoot meristems and in leaves, and what mechanisms stabilize "organizing auxin maxima." Future research will also have to point out whether specific PIN polarization rules, which could be set by molecular switches such as the PINOID kinase (Friml et al. 2004), are under developmental-context specific control of transcription factors to generate variants on an underlying theme of pattern formation in plants.

\section{Conclusion}

The paper of Scarpella et al. (2006) in this issue of Genes \& Development provides strong experimental support for the role of polar PIN1 expression and auxin flow in the selection of pre-procambial cells, a characteristic feature of the canalization concept. Furthermore, the observations explain closed venation networks and the phenotypes of vein continuity mutants. Moreover, comparison of the current information and modeling efforts in plant venation with analysis of leaf phyllotaxis and root development should reveal whether a unification of mechanisms establishing auxin maxima as drivers of pattern formation in plants is possible.

\section{References}

Aida, M., Beis, D., Heidstra, R., Willemsen, V., Blilou, I., Galinha, C., Nussaume, L., Noh, Y.S., Amasino, R., and Scheres, B. 2004. The PLETHORA genes mediate patterning of the Arabidopsis root stem cell niche. Cell 119: 109-120.

Aloni, R., Schwalm, K., Langhans, M., and Ullrich, C.I. 2003. Gradual shifts in sites of free-auxin production during leafprimordium development and their role in vascular differentiation and leaf morphogenesis in Arabidopsis. Planta 216: 841-853.

Benkova, E., Michniewicz, M., Sauer, M., Teichmann, T., Seifertova, D., Jurgens, G., and Friml, J. 2003. Local, efflux-dependent auxin gradients as a common module for plant organ formation. Cell 115: 591-602.

Berleth, T. 2000. Plant development: Hidden networks. Curr. Biol. 10: R658-R661.

Blilou, I., Xu, J., Wildwater, M., Willemsen, V., Paponov, I., Friml, J., Heidstra, R., Aida, M., Palme, K., and Scheres, B. 2005. The PIN auxin efflux facilitator network controls growth and patterning in Arabidopsis roots. Nature 433: 3944.

Carland, F.M., Berg, B.L., FitzGerald, J.N., Jinamornphongs, S., Nelson, T., and Keith, B. 1999. Genetic regulation of vascular tissue patterning in Arabidopsis. Plant Cell 11: 21232137.

de Reuille, P.B., Bohn-Courseau, I., Ljung, K., Morin, H., Carraro, N., Godin, C., and Traas, J. 2006. Computer simulations reveal properties of the cell-cell signaling network at the shoot apex in Arabidopsis. Proc. Natl. Acad. Sci. 103: $1627-1632$.

Deyholos, M.K., Cordner, G., Beebe, D., and Sieburth, L.E. 2000. The SCARFACE gene is required for cotyledon and leaf vein patterning. Development 127: 3205-3213.

Friml, J., Benkova, E., Blilou, I., Wisniewska, J., Hamann, T., Ljung, K., Woody, S., Sandberg, G., Scheres, B., Jurgens, G., et al. 2002. AtPIN4 mediates sink-driven auxin gradients and root patterning in Arabidopsis. Cell 108: 661-673.

Friml, J., Vieten, A., Sauer, M., Weijers, D., Schwarz, H., Hamann, T., Offringa, R., and Jurgens, G. 2003. Efflux-de- 
pendent auxin gradients establish the apical-basal axis of Arabidopsis. Nature 426: 147-153.

Friml, J., Yang, X., Michniewicz, M., Weijers, D., Quint, A., Tietz, O., Benjamins, R., Ouwerkerk, P.B., Ljung, K., Sandberg, G., et al. 2004. A PINOID-dependent binary switch in apical-basal PIN polar targeting directs auxin efflux. Science 306: $862-865$.

Gälweiler, L., Guan, C., Müller, A., Wisman, E., Mendgen, K., Yephremov, A., and Palme, K. 1998. Regulation of polar auxin transport by AtPIN1 in Arabidopsis vascular tissue. Science 282: 2226-2230.

Geldner, N., Anders, N., Wolters, H., Keicher, J., Kornberger, W., Muller, P., Delbarre, A., Ueda, T., Nakano, A., and Jurgens, G. 2003. The Arabidopsis GNOM ARF-GEF mediates endosomal recycling, auxin transport, and auxin-dependent plant growth. Cell 122: 219-230.

Heisler, M.G., Ohno, C., Das, P., Sieber, P., Reddy, G.V., Long, J.A., and Meyerowitz, E.M. 2005. Patterns of auxin transport and gene expression during primordium development revealed by live imaging of the Arabidopsis inflorescence meristem. Curr. Biol. 15: 1899-1911.

Jonsson, H., Heisler, M.G., Shapiro, B.E., Meyerowitz, E.M., and Mjolsness, E. 2006. An auxin-driven polarized transport model for phyllotaxis. Proc. Natl. Acad. Sci. 103: 1633-1638.

Koizumi, K., Sugiyama, M., and Fukuda, H. 2000. A series of novel mutants of Arabidopsis thaliana that are defective in the formation of continuous vascular network: Calling the auxin signal flow canalization hypothesis into question. Development 127: 3197-3204.

Koizumi, K., Naramoto, S., Sawa, S., Yahara, N., Ueda, T., Nakano, A., Sugiyama, M., and Fukuda, H. 2005. VAN3 ARFGAP-mediated vesicle transport is involved in leaf vascular network formation. Development 132: 1699-1711.

Mattsson, J., Sung, Z.R., and Berleth, T. 1999. Responses of plant vascular systems to auxin transport inhibition. Development 126: 2979-2991.

Meinhardt, H. 1976. Morphogenesis of lines and nets. Differentiation 6: 117-123.

. 1984. Models of pattern formation and their application to plant development. In Positional controls in plant development (eds. P.W. Barlow and D.J. Carr), pp. 1-32. Cambridge University Press, Cambridge.

Meinhardt, H., Koch, A.J., and Bernasconi, G. 1998. Models of pattern formation applied to plant development. In Symmetry in plants (eds. D. Barabe and R.V. Jean), pp. 723-758. World Scientific Publishing, Singapore.

Mitchison, G.J. 1980. Model for vein formation in higher-plants. Proc. R. Soc. Lond. B. Biol. Sci. 207: 79-109.

Reinhardt, D., Mandel, T., and Kuhlemeier, C. 2000. Auxin regulates the initiation and radial position of plant lateral organs. Plant Cell 12: 507-518.

Reinhardt, D., Pesce, E.R., Stieger, P., Mandel, T., Baltensperger, K., Bennett, M., Traas, J., Friml, J., and Kuhlemeier, C. 2003. Regulation of phyllotaxis by polar auxin transport. Nature 426: $255-260$.

Rolland-Lagan, A.G. and Prusinkiewicz, P. 2005. Reviewing models of auxin canalization in the context of leaf vein pattern formation in Arabidopsis. Plant J. 44: 854-865.

Sabatini, S., Beis, D., Wolkenfelt, H., Murfett, J., Guilfoyle, T., Malamy, J., Benfey, P., Leyser, O., Bechtold, N., Weisbeek, P., et al. 1999. An auxin-dependent distal organizer of pattern and polarity in the Arabidopsis root. Cell 99: 463-472.

Sachs, T. 1981. The control of the patterned differentiation of vascular tissues. Adv. Bot. Res. 9: 151-262.

. 1991. Cell polarity and tissue patterning in plants. Development 91 (Suppl.): 83-93.
Scarpella, E., Francis, P., and Berleth, T. 2004. Stage-specific markers define early steps of procambium development in Arabidopsis leaves and correlate termination of vein formation with mesophyll differentiation. Development 131: 3445-3455.

Scarpella, E., Marcos, D., Friml, J., and Berleth, T. 2006. Control of leaf vascular patterning by polar auxin transport. Genes \& Dev. (this issue).

Scheres, B. and Berleth, T. 1998. Root development: New meanings for root canals? Curr. Opin. Plant Biol. 1: 32-36.

Sieburth, L.E. 1999. Auxin is required for leaf vein pattern in Arabidopsis. Plant Physiol. 121: 1179-1190.

Smith, R.S., Guyomarch, S., Mandel, T., Reinhardt, D., Kuhlemeier, C., and Prusinkiewicz, P. 2006. A plausible model of phyllotaxis. Proc. Natl. Acad. Sci. 103: 1301-1306.

Turing, A. 1952. The chemical basis of morphogenesis. Philos. Trans. R. Soc. Lond. B Biol. Sci. 237: 37-72.

Vieten, A., Vanneste, S., Wisniewska, J., Benkova, E., Benjamins, R., Beeckman, T., Luschnig, C., and Friml, J. 2005. Functional redundancy of PIN proteins is accompanied by auxin-dependent cross-regulation of PIN expression. Development 132: 4521-4531.

Wisniewska, J., Xu, J., Seifertová, D., Brewer, P.B., Ruzicka, K., Blilou, I., Benková, E., Scheres, B., and Friml, J. 2006. Polar PIN localization is sufficient to direct auxin flow in plants. Science (in press).

Xu, J. and Scheres, B. 2005. Dissection of Arabidopsis ADPRIBOSYLATION FACTOR 1 function in epidermal cell polarity. Plant Cell 17: 525-536.

$\mathrm{Xu}$, J., Hofhuis, H., Heidstra, R., Sauer, M., Friml, J., and Scheres, B. 2006. A molecular framework for plant regeneration. Science 311: 385-388. 


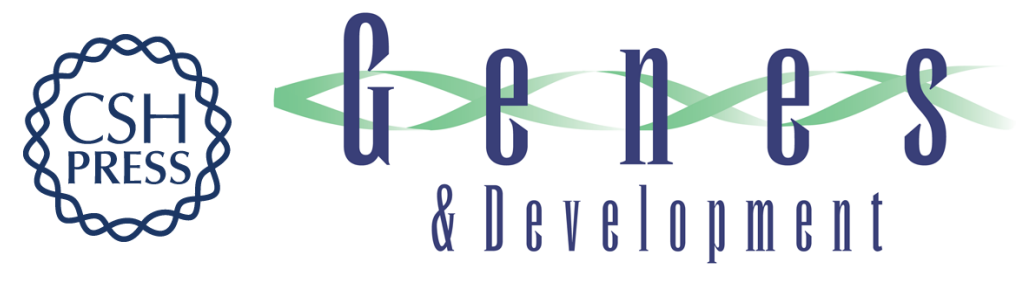

\section{Polar auxin transport and patterning: grow with the flow}

Ben Scheres and Jian Xu

Genes Dev. 2006, 20:

Access the most recent version at doi:10.1101/gad.1426606

Related Content Control of leaf vascular patterning by polar auxin transport Enrico Scarpella, Danielle Marcos, Jirí Friml, et al. Genes Dev. UNKNOWN , 2006 20: 1015-1027

References This article cites 34 articles, 16 of which can be accessed free at: http://genesdev.cshlp.org/content/20/8/922.full.html\#ref-list-1

Articles cited in:

http://genesdev.cshlp.org/content/20/8/922.full.html\#related-urls

\section{License}

Email Alerting

Receive free email alerts when new articles cite this article - sign up in the box at the top Service right corner of the article or click here.

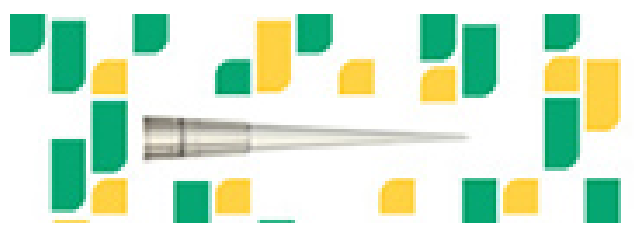

Focused on your science. 